\title{
Casa Melnikov. Racionalismo en la Unión Soviética
}

\section{Ramiro Pascal de Marzo}

Universidad de Lima, Perú

Recibido: 16 de mayo de 2014 / Aprobado: 11 de julio de 2014

La obra de Konstantine Melnikov es una vivienda representativa del siglo XX, donde se ven las ideas del espacio moderno combinadas con las limitaciones técnicas, que se adaptan a un contexto de crisis y escasez como fue la Unión Soviética a mediados del siglo XX. La singular geometría y las posibilidades de la construcción artesanal, pero pensada desde la fabricación en serie, así como el desarrollo del espacio vertical, hacen de esta obra una de las más importantes construcciones de la arquitectura moderna europea. El análisis aborda estas variables, y busca plantear su importancia como materia de estudio.

arquitectura, Melnikov, Unión Soviética, vivienda, arquitectura moderna, siglo XX

\section{Melnikov House. Rationalism in the Soviet Union}

Konstantine Melnikov's work is a representative apartment in the twentieth century, where the combination between the ideas of modern space combined with the limited techniques that adapt to a crisis and scarcity as the Soviet Union was at the mid-twentieth century. The unique geometry and possibilities of craft construction but designed from mass production, and the development of vertical space make this work one of the most important buildings of modern european architecture. The analysis addresses these variables, and seeks to raise its importance as a subject of study.

architecture, Melnikov, Soviet Union, house, modern architecture, 20th century 

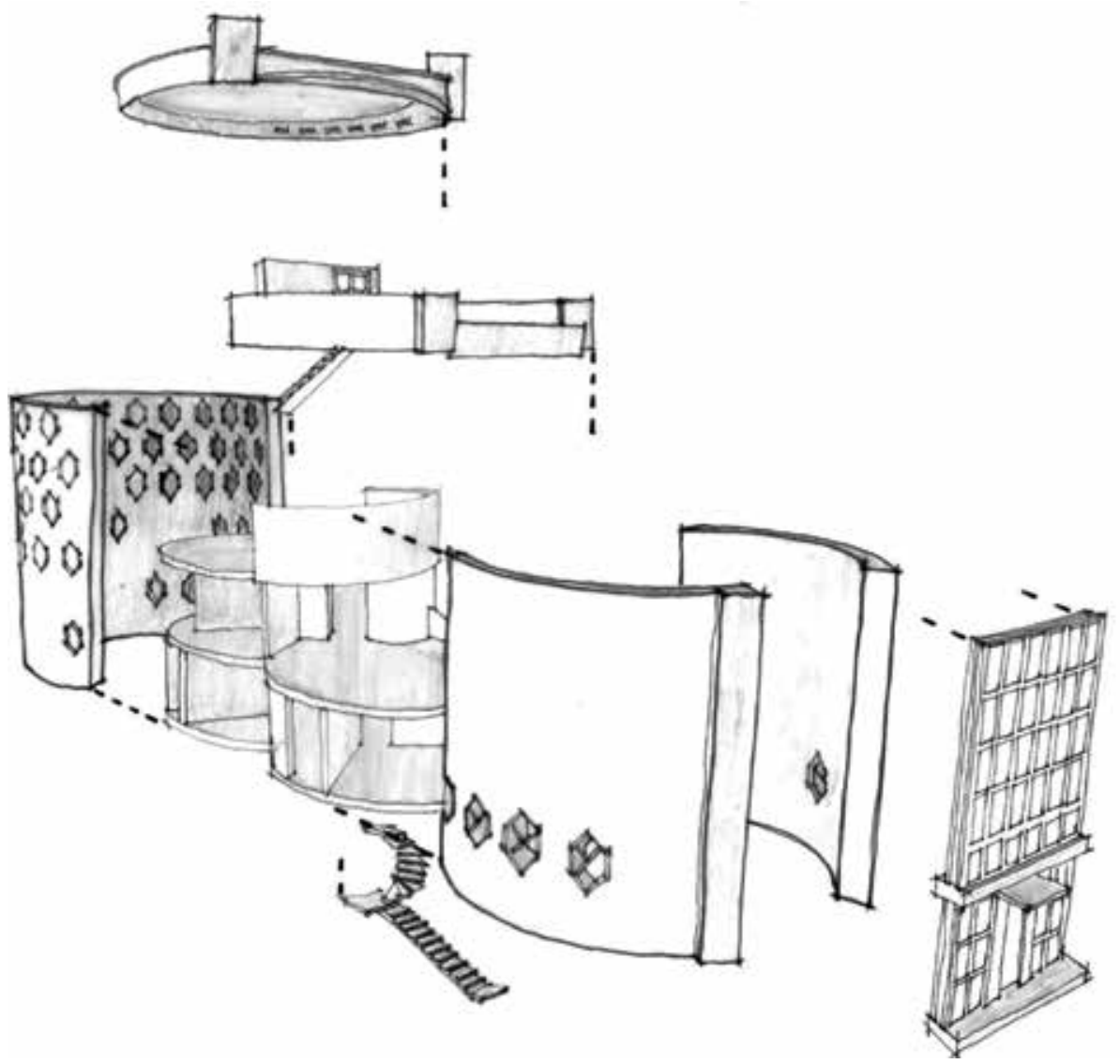

Figura1.

Axonometría explotada.

\section{INTRODUCCIÓN}

Me interesó mucho analizar este proyecto, porque marca un hito en el modernismo ruso debido a que se desarrolló entre dos periodos muy complicados en la historia de Rusia: primero, un contexto previo de interrupción del ciclo ordinario en cuanto al desarrollo arquitectónico y de construcción, causado por las guerras civiles, la primera y segunda guerra mundial, las revoluciones sociales y militares, y la crisis de la posguerra;segundo, un contexto posterior, cuando Stalin sube al poder, quien con un carácter totalitario y absoluto decide dibujarle un nuevo rostro al país, teniendo como pilar la arquitectura monumental y neoclásica: la arquitectura del nuevo régimen, que fomentó la colectivización, en la cual la casa Melnikov, que comenzó como modelo experimental, purista y casi abstracto, terminó siendo considerada como una perversión por el régimen de Stalin, porque iba en contra de sus ideas, de "la nueva cara de Rusia". Esto fue motivo para que a Konstantine Melnikov se le quitara el derecho de enseñar y hacer arquitectura, además de ser condenado a vivir bajo arresto domiciliario, pasando el resto de sus años ganándose la vida como artista.

La casa Melnikov, la obra más famosa de este arquitecto, con su revolucionaria forma y original concepción, significó el antes y el después de la vida de un arquitecto.

\section{ANÁLISIS CRÍTICO}

El edificio es de forma simple y estética pura, Melnikov renuncia totalmente al ornamento y 


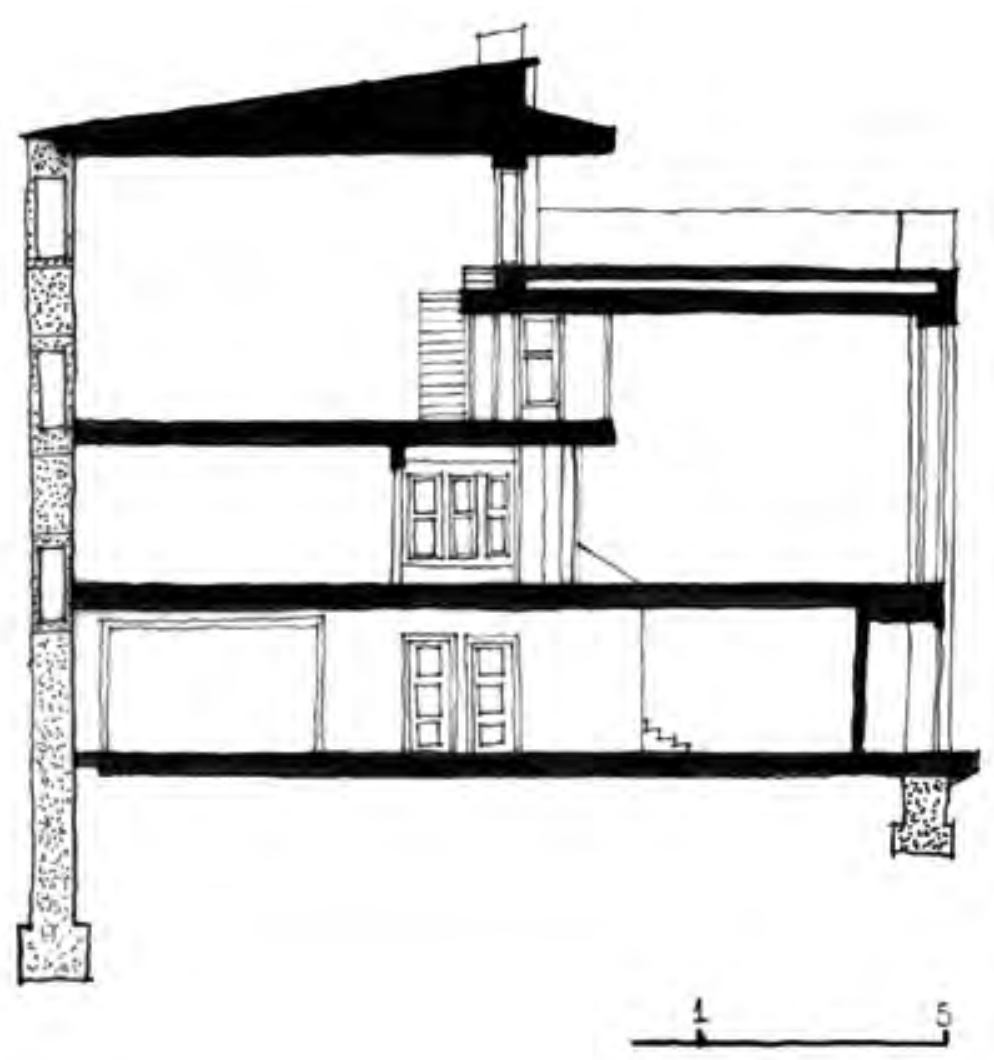

Figura 2.

Sección.

diseña su casa de manera limpia, compuesta por dos cilindros blancos de nueve metros de diámetro que se entrelazan en un eje longitudinal para buscar la simetría en planta; del mismo modo, los espacios se entrelazan en sección para lograr espacios de doble altura. Tiene una gran fachada de vidrio enmarcada por dos columnas cuadradas; al enmarcar la entrada principal vemos que el arquitecto aún tiene influencia de la arquitectura clásica.

Esta gran fachada es una característica importante de la casa, ya que es el frente principal compuesta de vidrio y marcos de metal negro, que permite una gran iluminación al espacio del salón principal y al estudio de Melnikov, es decir, el trabajo de fachada exterior refleja la importancia del espacio interior, característico del modernismo. En general, la casa cuenta con una remarcable iluminación debido a la homogénea distribución de las 59 ventanas hexagonales, que iluminan todos los ambientes de la casa, dando un lenguaje exterior muy simple a modo de piel, que con cinco niveles horizontales confunden e impiden distinguir el número de pisos de la casa y distorsionan la percepción de la escala.

Debido a la escasez de recursos, Melnikov usó el material más económico: el ladrillo. La casa se estructura con forjados reticulares ligeros de madera laminada y muros de carga gruesos perimetrales de ladrillo y la carga es transmitida a través de los arcos intercalados que forman las ventanas hexagonales, hacia los cimientos de escombros apisonados.

Cuando estaba terminado el perímetro de ladrillo perforado homogéneamente, Melnikov 




Figura 3.

Planta primer nivel.

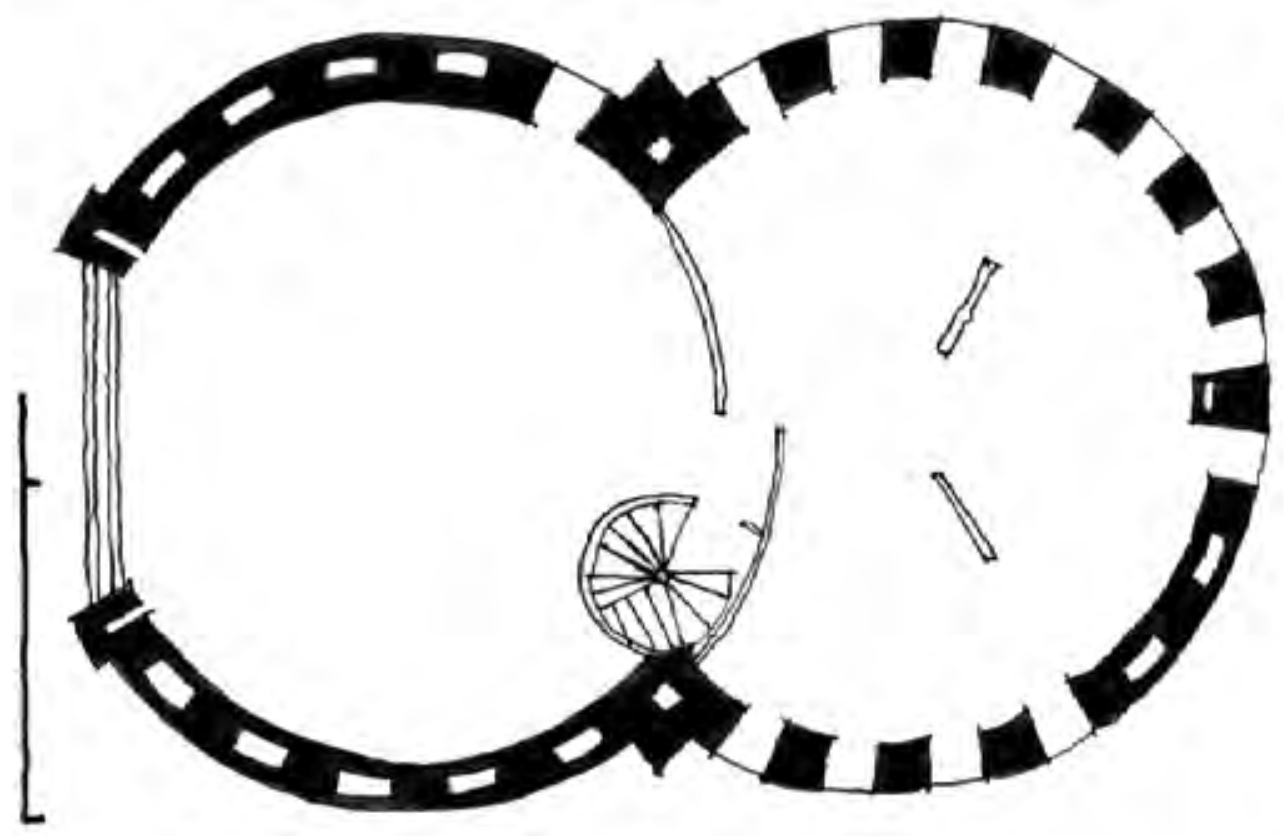

Figura 4.

Planta segundo nivel.

Limaq N. 12015 ISSN: 2410-6127 


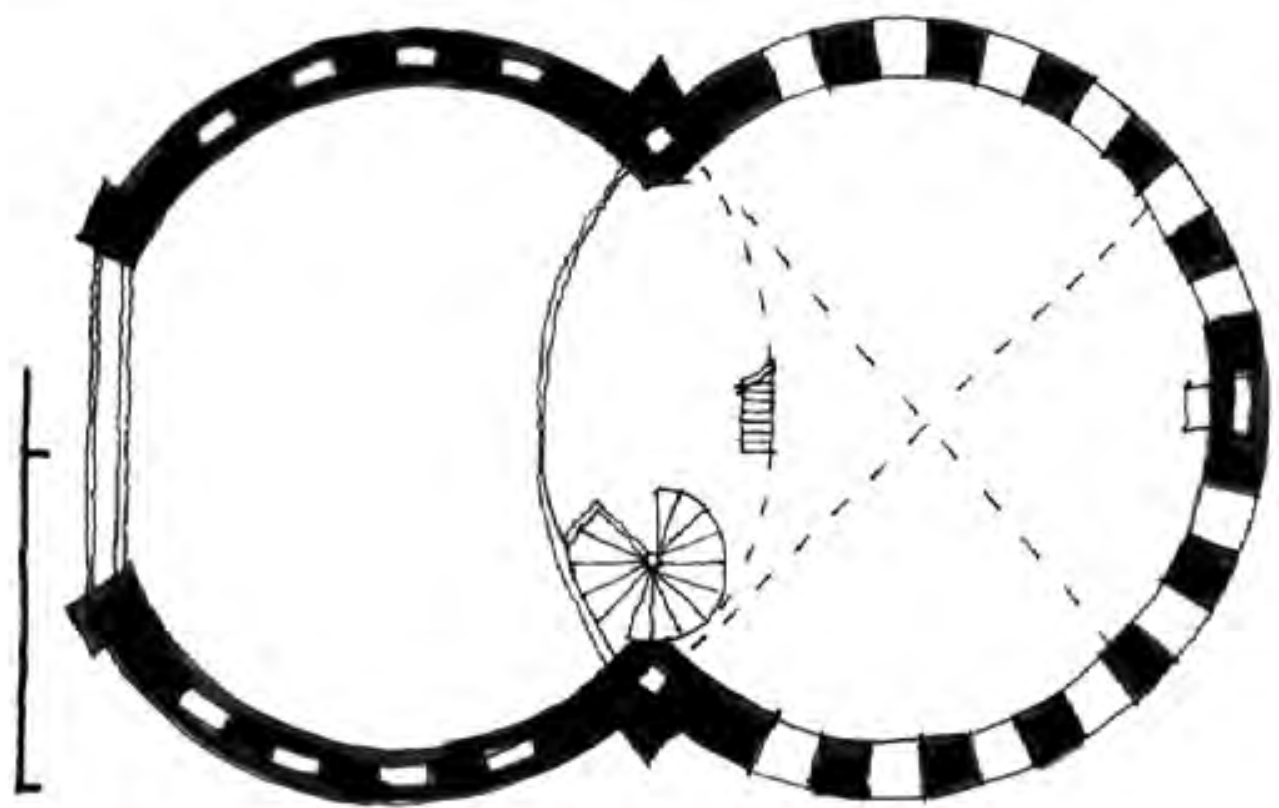

Figura 5.

Planta tercer nivel.

decidía qué perforaciones cubrir, con escombros, y qué perforaciones dejar abiertas como ventanas; finalmente, el edificio era recubierto en su totalidad con hormigón blanco, haciendo de esta manera más homogénea la casa, sin el caos o desorden que para él suponía la estructura de ladrillos. Además, usa el sistema de doble vidrio con una cámara de aire de al menos 15 centímetros para el aislamiento térmico de la casa.

Comodistribución espacialdestacasu funcionalismo e innovación. A pesar de la aparente limpieza de la forma y la simetría de los cilindros a través del eje longitudinal, el interior es más complejo y no siempre responde a esta simetría, ya que los espacios atraviesan oblicuamente dicho eje, dando prioridad a la distribución eficiente de los espacios. Las escaleras están colocadas en las intersecciones de los dos cilindros que permiten observar las habitaciones superiores de forma oblicua y no frontalmente.

En el primer nivel de la casa se desarrollan las actividades de uso diario o living activities, en el primer cilindro de este nivel se ubican el vestíbulo, el comedor y la cocina. En el cilindro siguiente, al mismo nivel, el lavadero, el amplio vestidor, una pequeña sala y el baño. Ambos cilindros se intersectan.

Para subir al segundo piso se debe recorrer una escalera recta de dos tramos para llegar a un hall muy pequeño, espacio intermedio entre las dos grandes estancias; la primera a la que se accede es a la gran sala de estar de doble altura, orientada a la calle y por tanto iluminada por el gran ventanal; en la siguiente estancia, en el 




Figura 6.

Planta cuarto nivel.

segundo cilindro, está el dormitorio, que ocupa todo el segundo cilindro y que en contraste con el gran salón es de techo bajo e iluminado con las pequeñas ventanas en forma de hexágono. Para acceder al tercer nivel, donde se ubica el estudio de Melnikov, se sube por una escalera de caracol; una vez más, usa toda una planta del cilindro, de aproximadamente $50 \mathrm{~m}^{2}$, de doble altura e iluminado por ventanas hexagonales. El estudio cuenta con una galería que comunica con una terraza, a la que se accede mediante una escalera de mano. La terraza está conectada visualmente con el estudio, y viceversa.

Todas estas condiciones de materialidad, distribución espacial, forma, relación de fachada con espacio interior, iluminación e innovación constructiva, hacen de la casa Melnikov una obra de la arquitectura moderna.

\section{CONCLUSIONES}

En esta época de cambios y revoluciones en la industria, se dio paso a la creación de un tipo particular de arquitectura: la vivienda obrera; sus características fueron su economicidad, su rápida construcción y su eficiencia espacial, y el estar bien distribuidos, a manera de módulo, que generaron los barrios obreros.

La casa Melnikov también fue importante porque en sus inicios fue desarrollada como proyecto social, por ello llegó a ser económica, flexible, eficiente, sostenible y de rápida y fácil construcción, pues fue pensada como vivienda obrera; y terminó convirtiéndose en un destacado hito del modernismo ruso, por su estereotomía y simpleza de la forma, con una disposición original, el manejo y la importancia 
de la luz, con una vocación doméstica y distribución racionalista de los espacios, pensando siempre en el confort del usuario durante el transcurso del día.

Pero en su época, durante la Unión Soviética de Stalin, fue considerada una aberración, lo que significó el fin de la vida de Konstantine Melnikov como arquitecto; sin embargo ahora es vista como un icono del constructivismo ruso. Es el reflejo del pensamiento atemporal de un artista, que se mantiene hasta el día de hoy como modelo y símbolo de arquitectura pura y libre.

\section{REFERENCIAS}

Asensio, A. (2013). Una vida / La Casa Mellnikov. Plataforma Arquitectura. Recuperado de :http:// www.plataformaarquitectura.cl/2013/04/27/unavida-la-casa-melnikov/

J.-L. (2013). Videolecture, iconic Houses/Melnikov House. Recuperado de: http://www.youtube. $\mathrm{com} /$ watch? $\mathrm{v}=9 \mathrm{z} 3 \mathrm{AQQ} 1 \mathrm{ZBxaM}$

Khan-Magomedov, S.O. (2014).Konstantine Melnikov. Melnikov House. Recuperado de http://www. melnikovhouse.org/about-melnikov.php 